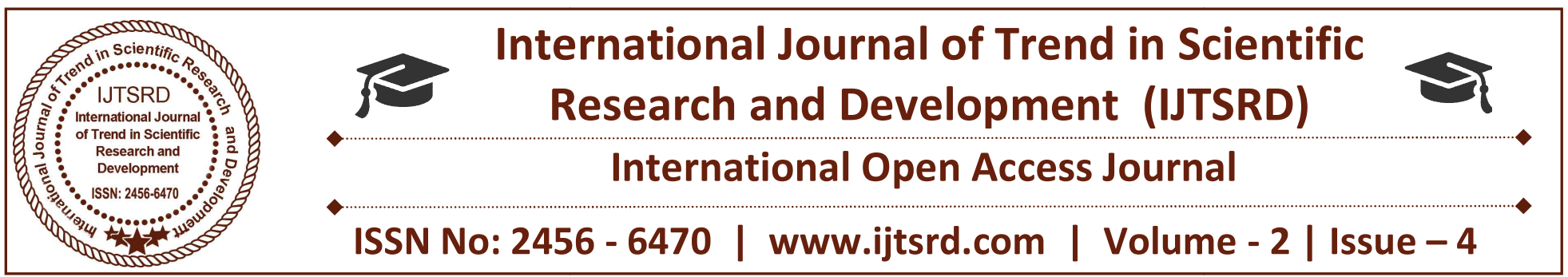

\title{
Involvement of Adolescents in Armed Conflict
}

\author{
Shazia Manzoor ${ }^{1}$, Dr. Muzamil Jan ${ }^{2}$ \\ ${ }^{1}$ Research Scholar, ${ }^{2}$ Sr. Assistant Professor \\ Institute of Home Science, Kashmir University, Srinagar, Jammu Kashmir, India
}

\begin{abstract}
The present study was an attempt to observe the involvement of adolescents in Kashmir conflict as per affect of conflict (intact and disrupted), dwelling and gender. The sample was selected through multi stage sampling technique. For the sample 800 adolescents were selected from urban and rural areas of Kashmir. The sample was collected with the help of self constructed questionnaire. The study reveals that most intact and disrupted adolescents have participated in protests. More adolescent boys have participated in protests as compared to adolescent girls. Greater number of intact rural adolescents has participated in protests as compared to intact urban adolescents. Majority of adolescents have participated more than once. Causes put forth for participation in protests by intact-disrupted adolescents, adolescent boys-girls and rural-urban adolescents are innocent killings, harassment of women and unjustified arrests of youth.
\end{abstract}

Keywords: Protests, reasons, armed conflict, Kashmir

\section{INTRODUCTION}

The world as a whole has been getting incrementally less peaceful every year since 2007 and bucking a trend that had seen a global move away from conflict since the end of the Second World War (Withnall, 2014). As per Global Peace Index 2017, most peaceful countries are Ice land, New Zealand, Portugal, Austria, Denmark, Czech Republic, Slovenia, Canada, Switzerland and Ireland. While as least peaceful nations in world are Syria, Afghanistan, Yemen, Somalia, Libya, Sudan, Central African Republic, Ukraine, Democratic Republic of Congo, Pakistan and North Korea (IEP, 2017). Adolescents today are increasingly deliberate targets, as well as unintended victims in armed conflicts around the world. Between 1985-1995 two million children were killed, six million were left seriously injured or permanently disabled, twelve million were left homeless, one million were orphaned or separated from their parents, ten million suffered from serious psychological trauma as a result of war and 300000 served as child soldiers (Gow et al, 2000). During 2016 unrest in Kashmir 96 people were reported to have died and 5,000-6,000 adults and children were injured. Children as young as four and five years had multiple pellets in their retinas, blinding them partially or completely, for life. These patients were brought to hospitals with serious injuries, including blinding and other permanent disabilities caused by the pellets. Amputations, permanent disabilities and the loss of life affected thousands who occupied the streets and were often not in any way engaged in the conflict. Even those on the sidelines or watching the confrontations from their homes as mere observers were hit by pellets (Nadimpally, 2017).

\section{REVIEW of literature}

Amone-P'olak et al (2007) assessed war experiences and the impact of physical abuse on formerly abducted boys in Northern Uganda. Cross sectional self report designs were used on 216 formerly abducted boys and it was found that all boys were exposed to war events, participated in violence, were bodily abused and manifested numerous signs and symptoms of post traumatic stress.

Okello et al (2007) conducted a cross sectional study on war abducted and non abducted adolescents in Northern Uganda to assess nature and patterns of psychiatric disorders. Elevated occurrence of major depression, generalized anxiety disorder, post 
traumatic stress disorder and co morbidity was found in war abducted adolescents in contrast to nonabducted adolescents.

Betancourt et al (2012) conducted a study to explore linked with internalizing emotional and behavioral problems among adolescents dislocated during the most recent Chechen conflict and showed that levels of internalizing problems were higher in dislocated Chechen youth compared to non referred youth in United States and among Russian children not affected by conflict. Girls demonstrated higher problems scores compared to boys.

Khamis (2012) observed 600 adolescent participants from Gaza strip and South Lebanon aged 12 to 16 years to explore differences in the types of war trauma, economic pressure religiosity and psychiatric disorders, interviews were done through questionnaires by trained psychologists and results indicated that adolescents who were under great economic suffering were at the maximum risk for psychological grief including PTSD, depression and anxiety. Contrary to adolescents from South Lebanon, higher levels of religiosity in Gazans indicated the top levels of depression and anxiety. While ideology was negatively related with depression and anxiety in Gazans, ideology did not play a role among adolescents from South Lebanon.

\section{OBJECTIVES}

The present study is based on the following objectives:

1. To study the involvement of adolescents in Kashmir conflict

2. To assess causes and frequency of participation of adolescents in Kashmir conflict.

\section{MATERIAL AND METHODS}

The present study was an attempt to study the involvement of adolescents in Kashmir conflict as per affect of conflict (intact and disrupted), dwelling and gender. The information was gathered from adolescents in urban and rural areas of Kashmir region. The study was investigated through multi stage sampling technique. This type of sampling was taken because the size of population (i.e. Kashmir) was very large and was scattered as per socioeconomic characteristics of adolescents. The sample selected by this method was more representative of population. It permitted the fieldwork to be concentrated and yet large area covered. Sample was selected from Kashmir region-800 adolescents were selected for the purpose, 400 adolescents from urban areas and 400 adolescents from rural areas. The tool used for the study includes a detailed questionnaire. The data obtained was carefully scrutinized, categorized and coded in order to fulfill the objectives. The data was analyzed applying appropriate statistical measures.

\section{RESULTS AND DISCUSSION}

\section{Involvement of Adolescents in Conflict:}

There is a growing trend of adolescent's participation in protests in Kashmir. Since 2010 adolescents usually remain on the front lines of protest, lead demonstrations, and use social media as a foremost mode of their expression.

\section{Participation in Protests}

With each passing day Kashmir issue becomes tenser and each uprising witness new trends of revolt. For this reason table 4.1 reveals that 70.5 per cent intact and 50.5 per cent $(\mathrm{f}=101)$ disrupted adolescents have participated in protests. More adolescent boys i.e.75.25 per cent $(\mathrm{f}=301)$ have participated in protests as compared to 59.75 per cent $(f=239)$ adolescent girls. However, participation of rural and urban adolescents is almost same i.e.69 per cent $(\mathrm{f}=276)$ rural and 66 per cent $(\mathrm{f}=264)$ urban respectively. Participation in protests show highly significant differences among intact-disrupted adolescents $\chi^{2}$ $(1,800)=16.7, \mathrm{p}=0.00$ and adolescent boys-girls $\chi^{2}$ $(1,800)=21.90, \quad \mathrm{p}=0.00 ;$ while rural-urban adolescents show insignificant differences $\chi^{2}(1,800)$ $=0.82, \mathrm{p}=0.36$. Involvement in protests reveals negative highly significant correlation among intactdisrupted adolescents $\mathrm{r}(800)=-0.149, \mathrm{p}=0.000$ and positive highly significant correlation between adolescent boys-girls $\mathrm{r}(800)=0.165, \mathrm{p}=0.000$. However, positive insignificant correlation is observed among rural-urban adolescents $\mathrm{r}(800)$ $=0.032, \mathrm{p}=0.366$.

Table 4.2 shows that most intact rural adolescents i.e.63 per cent $(\mathrm{f}=126)$ have participated in protests as compared to 42 per cent $(\mathrm{f}=84)$ intact urban adolescents who have never participated. In contrast to this, 75 per cent $(\mathrm{f}=150)$ disrupted rural adolescents and 74 per cent $(\mathrm{f}=148)$ disrupted urban adolescents have also participated in protests. Involvement in protests show insignificant differences among intact rural-urban adolescents $\chi^{2}(1,400)=1.04, p=0.30$ and disrupted rural-urban adolescents $\chi^{2}(3,400)=0.053$, 
$\mathrm{p}=0.817$. In this context, negative significant correlation is seen among intact rural-urban adolescents $\mathrm{r}(400)=0.130, \mathrm{p}=0.009$ and negative highly significant correlation is observed among disrupted rural-urban adolescents $\mathrm{r}(400)=-0.169$, $\mathrm{p}=0.001$.

Table 4.3 presents that a good number of intact boys i.e.70.5 per cent $(\mathrm{f}=141)$ have participated in protests; while as only 50.5 per cent ( $\mathrm{f}=101)$ intact girls have participated in protests. In the same manner, 80.5 per cent $(\mathrm{f}=160)$ disrupted boys have participated in protests and in comparison to that only 69 per cent $(\mathrm{f}=138)$ disrupted girls have participated. Highly significant differences are seen among intact boysgirls associated to participation in protests $\chi^{2}(1,400)$ $=16.7, \mathrm{p}=0.00$. However disrupted boys-girls show insignificant differences $\chi^{2}(1,400)=6.36, p=0.11$ in this context.

Mirani (2016) observed that almost every day a group of children between the age group 4-9 years in a safer locality of Srinagar comes out chanting slogans. For hours they go on sloganeering and marching from one end of the colony to another and this activity still continues. Mehta (2016) found that in 2016 unrest young people, who constitute the largest chunk of Kashmir's population, took to throwing stones in protest on the streets and used social media to tell their stories. Demonstrations continued despite heavy security presence and shutdown of mobile internet and phone services. Malik (2016) stated that a rapid mobilization process of women for the cause of resistance, attracting huge number of middle-class urban women, college and university girl students, female academicians and professionals, happened in 2008 when millions of Kashmiri people came out on the streets and peacefully demonstrated against land transfer.

\section{Frequency of Participation}

Table 4.1 put forth that among intact adolescents, 35.5 per cent $(\mathrm{f}=50)$ always participate in protests; while as 45.5 per cent $(\mathrm{f}=46)$ disrupted adolescents have participated only once. Similarly, 33.88 ( $\mathrm{f}=102)$ boys have always participated in protests, whereas 41.84 per cent $(\mathrm{f}=100)$ have participated just once. On contrary to this, 40.21 per cent $(\mathrm{f}=111)$ rural and 37.12 per cent $9 \mathrm{f}=98$ ) urban adolescents have participated once. On the whole, 39.1 per cent (54) adolescents have participated just once. About 24.6 per cent $(\mathrm{f}=34)(\mathrm{f}=24)$ have participated sometimes,
18.8 per cent $(\mathrm{f}=26)$ have always participated and 17.4 per cent $(\mathrm{f}=24)$ have participated often in protests. In relation to rate of participation in protests, highly significant differences are seen among intactdisrupted adolescents $\chi^{2}(3,800)=29.8, p=0.00$ and adolescent boys-girls $\chi^{2}(3,800)=42.31, \mathrm{p}=0.00$. However rural-urban adolescents show insignificant differences $\chi^{2}(3,800)=2.04, p=0.56$. Extent in participation depicts negative insignificant correlation between intact-disrupted adolescents $\mathrm{r}(800)=-0.004$, $\mathrm{p}=0.932$; positive highly significant correlation between adolescent boys-girls $r(800)=-0.176$, $\mathrm{p}=0.000$ and positive insignificant correlation among rural-urban adolescents $\mathrm{r}(800)=0.043, \mathrm{p}=0.323$.

Table 4.2 reveals that among rural intact adolescents, 42.1 per cent $(\mathrm{f}=53)$ have participated only once and 26.2 per cent $(\mathrm{f}=33)$ participated sometimes. Similarly 32.8 per cent $(\mathrm{f}=38)$ urban adolescents have participated once and 31.9 per cent $(\mathrm{f}=37)$ participated sometimes. In the same way, 27.3 per cent $9 f=41$ ) disrupted rural and 25 per cent $(\mathrm{f}=37)$ urban adolescents have always participated even though 38.7 per cent $(\mathrm{f}=58)$ disrupted rural and 40.5 per cent $(\mathrm{f}=60)$ disrupted urban have participated just once. Insignificant differences are evident among intact rural-urban adolescents $\chi^{2}(3,400)=4.65, \mathrm{p}=0.30$ and disrupted rural-urban adolescents $\chi^{2}(3,400)=0.41$, $\mathrm{p}=0.93$. Rate of involvement in protests depicts positive insignificant correlation between intact ruralurban adolescents $\mathrm{r}(400)=0.064, \mathrm{p}=0.290$ and negative insignificant correlation between disrupted rural-urban adolescents $\mathrm{r}(400)=-0.076, \mathrm{p}=0.217$.

Table 4.3 specifies that quantity of participation is greater among boys as 35.5 per cent $(\mathrm{f}=50)$ and 27 per cent $(\mathrm{f}=38)$ intact boys participate always and sometimes respectively as compared to 45.5 per cent $(\mathrm{f}=460)$ who have participated only once even though 31.7 per cent $(\mathrm{f}=32)$ participate sometimes. In contrast to this, 40 per cent $(\mathrm{f}=64)$ disrupted boys have participated once. However 32.5 per cent $(f=52)$ always participated in the protests. Similarly, 39.1 per cent $(f=54)$ disrupted girls have participated once while 24.6 per cent $(\mathrm{f}=34)$ have participated sometimes. Presence in protests reveal highly significant differences among intact boys-girls $\chi^{2}$ $(3,400)=29.8, \mathrm{p}=0.00$ and disrupted boys-girls $\chi^{2}$ $(3,400)=19.0, p=0.00$. Protest participation shows negative insignificant correlation between intact boys and intact girls $r(400)-0.2, p>0.05$; while, positive but insignificant correlation among disrupted boys and 
disrupted girls $r(400) 0.8, p>0.05$ are observed in this context.

Bazaz (2016) reported that the four districts of south Kashmir- Pulwama, Anantnag, Shopian and Kulgam remained under curfew for months together since the uprising began due to killings. Youth used to block all the main squares, lanes and alleys in these four districts. Demonstrations and protests were their daily activity.

\section{Reason for Participation}

Table 4.1 depicts that causes put forth for participation in protests by 63.1 per cent $(\mathrm{f}=89)$ intact adolescents, 86 per cent $(\mathrm{f}=79)$ disrupted adolescents, 58.1 per cent $(\mathrm{f}=175)$ boys, 72.38 per cent $(\mathrm{f}=173)$ girls, 74.63 per cent $(\mathrm{f}=206)$ rural adolescents and $53.78(\mathrm{f}=142)$ per cent urban adolescents are innocent killings, harassment of women and unjustified arrests of youth. Motivation for participation in protests founds highly significant differences among intact-disrupted adolescents $\chi^{2}$ $(3,800)=39.1, \quad \mathrm{p}=0.00$, adolescent boys-girls $\chi^{2}$ $(3,800)=50.54, p=0.00$ and rural-urban adolescents $\chi^{2}$ $(3,800)=28.06, p=0.00$. Drive for involvement reveals positive significant correlation between intactdisrupted adolescents $r(800)=-0.107, p=0.013$ as well as adolescent boys-girls $\mathrm{r}(800)=.146, \mathrm{p}=0.001$. On the other hand negative highly significant correlation between rural-urban adolescents $\mathrm{r}(800)=-0.200$, $\mathrm{p}=0.000$ are also observed.

Table 4.2 shows that defense put forward by 85.7 per cent $(\mathrm{f}=108)$ intact rural, 51.7 per cent $(\mathrm{f}=60)$ intact urban, 65.3 per cent $(\mathrm{f}=98)$ disrupted rural and 55.4 per cent $(\mathrm{f}=82)$ disrupted urban adolescents comprise innocent killings, harassment of women and unjustified arrest of youth. Explanation for protest participation shows highly significant differences between intact rural-urban adolescents $\chi^{2}(3,400)$ $=39.5, \mathrm{p}=0.00$; while insignificant differences are found among disrupted rural-urban adolescents $\chi^{2}$ $(3,400)=3.45, p=0.32$. Cause for participation shows negative highly significant correlation between intact rural-urban adolescents $\mathrm{r}(400)=-0.221, \mathrm{p}=0.000$ and negative insignificant correlation among disrupted rural-urban adolescents $\mathrm{r}(400)=-0.013, \mathrm{p}=0.831$.

Table 4.3 illustrates that excuses for protests given by 63.1 per cent $(\mathrm{f}=89)$ intact boys, 78.2 per cent $(\mathrm{f}=79)$ intact girls, 53.8 per cent $(\mathrm{f}=86)$ disrupted boys and 68.1 per cent $(\mathrm{f}=94)$ disrupted girls involves innocent killings, harassment of women and unjustified arrests of youth. With reference to reason for participation intact boys and intact girls show highly significant differences $\chi^{2}(3,400)=39.1, p=0.00$ along with disrupted boys and disrupted girls $\chi^{2}(3,400)=18.4$, $\mathrm{p}=0.00$. Reason for participation signifies positive insignificant correlation between intact boys and intact girls $\mathrm{r}(400) 0.4, \mathrm{p}>0.05$ and disrupted boys and disrupted girls $\mathrm{r}(400) 0.8, \mathrm{p}>0.05$.

Bleasdale (2013) stated that thousands of children are serving as soldiers in armed conflicts around the world. These boys and girls, some as young as 8 years old, serve in government forces and armed opposition groups. They may fight on the front lines, participate in suicide missions, and act as spies, messengers, or lookouts. Girls may be forced into sexual slavery. Many are abducted or recruited by force, while others join out of desperation, believing that armed groups offer their best chance for survival. According to Mustafa (2016) the students in Kashmir picks up a stone to bring a reaction from a system which according to them are responsible for raids, arrests, harassment, and humiliation on the streets. 
International Journal of Trend in Scientific Research and Development (IJTSRD) ISSN: 2456-6470

4.1 Involvement in Armed Conflict

\begin{tabular}{|c|c|c|c|c|c|c|c|c|c|c|c|c|c|c|}
\hline \multirow[t]{3}{*}{ Variable } & \multicolumn{4}{|c|}{ Category $(\mathrm{N}=800)$} & \multicolumn{4}{|c|}{ Gender $(\mathbf{N}=\mathbf{8 0 0})$} & \multicolumn{4}{|c|}{ Dwelling $(\mathrm{N}=800)$} & \multirow{2}{*}{\multicolumn{2}{|c|}{$\begin{array}{c}\text { All } \\
\text { adolescent } \\
\text { s } \\
(\mathbf{n}=\mathbf{8 0 0})\end{array}$}} \\
\hline & \multicolumn{2}{|c|}{$\begin{array}{c}\text { Intact } \\
\text { adolescent } \\
s(n=400)\end{array}$} & \multicolumn{2}{|c|}{$\begin{array}{l}\text { Disrupted } \\
\text { adolescent } \\
s(n=400)\end{array}$} & \multicolumn{2}{|c|}{$\begin{array}{c}\text { Adolescen } \\
\text { t boys } \\
(n=400)\end{array}$} & \multicolumn{2}{|c|}{$\begin{array}{c}\text { Adolescen } \\
\text { t girls } \\
(n=400)\end{array}$} & \multicolumn{2}{|c|}{$\begin{array}{c}\text { Rural } \\
\text { adolescent } \\
\text { s } \\
(n=400)\end{array}$} & \multicolumn{2}{|c|}{$\begin{array}{c}\text { Urban } \\
\text { adolescent } \\
S(n=400)\end{array}$} & & \\
\hline & $\mathrm{F}$ & $\%$ & F & $\%$ & F & $\%$ & $\mathrm{~F}$ & $\%$ & $\mathrm{~F}$ & $\%$ & F & $\%$ & F & $\%$ \\
\hline \multicolumn{15}{|c|}{ Participated in protests } \\
\hline Participated & 141 & 70.5 & 101 & 50.5 & $\begin{array}{c}30 \\
1\end{array}$ & $\begin{array}{c}75.2 \\
5\end{array}$ & $\begin{array}{c}23 \\
9\end{array}$ & $\begin{array}{c}59.7 \\
5\end{array}$ & 276 & 69 & 264 & 66 & 138 & 69.0 \\
\hline $\begin{array}{l}\text { Not } \\
\text { participated }\end{array}$ & 59 & 29.5 & 99 & 49.5 & 99 & $\begin{array}{c}24.7 \\
5\end{array}$ & $\begin{array}{c}16 \\
1\end{array}$ & $\begin{array}{c}40.2 \\
5\end{array}$ & 124 & 31 & 136 & 34 & 62 & 31.0 \\
\hline$\chi^{2}$ & \multicolumn{4}{|c|}{$16.7, \mathrm{df}=1, \mathrm{p}=0.00$} & \multicolumn{4}{|c|}{$21.90, \mathrm{df}=1, \mathrm{p}=0.00$} & \multicolumn{4}{|c|}{$0.82, \mathrm{df}=1, \mathrm{p}=0.36$} & & \\
\hline $\mathbf{r}$ & \multicolumn{4}{|c|}{$-0.149, p=0.000$} & \multicolumn{4}{|c|}{$0.165, \mathrm{p}=0.000$} & \multicolumn{4}{|c|}{$0.032, p=0.366$} & & \\
\hline \multicolumn{15}{|c|}{ Frequency of participation } \\
\hline Once & 45 & 31.9 & 46 & 45.5 & $\begin{array}{c}10 \\
9\end{array}$ & $\begin{array}{c}36.2 \\
1\end{array}$ & $\begin{array}{c}10 \\
0\end{array}$ & $\begin{array}{c}41.8 \\
4\end{array}$ & 111 & 40.21 & 98 & 37.12 & 54 & 39.1 \\
\hline Often & 8 & 5.7 & 16 & 15.8 & 14 & 4.65 & 40 & $\begin{array}{c}16.7 \\
3\end{array}$ & 31 & 11.23 & 23 & 8.71 & 24 & 17.4 \\
\hline Sometimes & 38 & 27.0 & 32 & 31.7 & 76 & $\begin{array}{c}25.2 \\
4\end{array}$ & 66 & $\begin{array}{c}27.6 \\
1\end{array}$ & 68 & 24.63 & 74 & 28.03 & 34 & 24.6 \\
\hline Always & 50 & 35.5 & 7 & 6.9 & $\begin{array}{c}10 \\
2\end{array}$ & $\begin{array}{c}33.8 \\
8\end{array}$ & 33 & $\begin{array}{c}13.8 \\
0\end{array}$ & 66 & 23.91 & 69 & 26.13 & 26 & 18.8 \\
\hline$\chi^{2}$ & \multicolumn{4}{|c|}{$29.8, \mathrm{df}=3, \mathrm{p}=0.00$} & \multicolumn{4}{|c|}{$42.31, \mathrm{df}=3, \mathrm{p}=0.00$} & \multicolumn{4}{|c|}{$2.04, \mathrm{df}=3, \mathrm{p}=0.56$} & & \\
\hline $\mathbf{r}$ & \multicolumn{4}{|c|}{$-0.004, p=0.932$} & \multicolumn{4}{|c|}{$-0.176, p=0.000$} & \multicolumn{4}{|c|}{$0.043, p=0.323$} & & \\
\hline \multicolumn{15}{|c|}{ Reason for participation } \\
\hline $\begin{array}{l}\text { Innocent } \\
\text { killings }\end{array}$ & 36 & 25.5 & 8 & 53 & 89 & $\begin{array}{c}29.5 \\
6\end{array}$ & 40 & $\begin{array}{c}16.7 \\
3\end{array}$ & 52 & 18.84 & 77 & 29.1 & 32 & 23.2 \\
\hline $\begin{array}{l}\text { Harassmen } \\
\mathrm{t} \text { of women }\end{array}$ & 0 & 0.0 & 13 & 4 & 4 & 1.32 & 23 & 9.62 & 7 & 2.53 & 20 & 7.57 & 10 & 7.2 \\
\hline $\begin{array}{l}\text { Unjustified } \\
\text { arrests of } \\
\text { youth }\end{array}$ & 16 & 11.3 & 1 & 17 & 33 & 10.9 & 3 & 1.25 & 11 & 3.98 & 25 & 9.4 & 2 & 1.4 \\
\hline $\begin{array}{l}\text { All of the } \\
\text { above }\end{array}$ & 89 & 63.1 & 79 & 86 & $\begin{array}{c}17 \\
5\end{array}$ & 58.1 & $\begin{array}{c}17 \\
3\end{array}$ & $\begin{array}{c}72.3 \\
8\end{array}$ & 206 & 74.63 & 142 & 53.78 & 94 & 68.1 \\
\hline$\chi^{2}$ & \multicolumn{4}{|c|}{$39.1, \mathrm{df}=3, \mathrm{p}=0.00$} & \multicolumn{4}{|c|}{$50.54, \mathrm{df}=3, \mathrm{p}=0.00$} & \multicolumn{4}{|c|}{$28.06, \mathrm{df}=3, \mathrm{p}=0.00$} & & \\
\hline $\mathbf{R}$ & \multicolumn{4}{|c|}{$-0.107, p=0.013$} & \multicolumn{4}{|c|}{$0.146, p=0.001$} & & -0.200 & $=0.0$ & & & \\
\hline
\end{tabular}

Based on field survey

df denotes degree of freedom

$\chi 2$ denotes chi square

$\mathrm{p}$ denotes spearman's level of significance

$\mathrm{r}$ denotes spearman's correlation 
Table 4.2 Involvement in Conflict as per Dwelling

\begin{tabular}{|c|c|c|c|c|c|c|c|c|}
\hline \multirow[t]{3}{*}{ Variable } & \multicolumn{4}{|c|}{ Intact $(\mathrm{N}=400)$} & \multicolumn{4}{|c|}{ Disrupted $(\mathrm{N}=400)$} \\
\hline & \multicolumn{2}{|c|}{ Rural } & \multicolumn{2}{|c|}{ Urban } & \multicolumn{2}{|c|}{ Rural } & \multicolumn{2}{|c|}{ Urban } \\
\hline & F & $\%$ & $\mathrm{~F}$ & $\%$ & $\mathrm{~F}$ & $\%$ & $\mathrm{~F}$ & $\%$ \\
\hline \multicolumn{9}{|l|}{ Participated in protests } \\
\hline Participated & 126 & 63.0 & 116 & 58.0 & 150 & 75.0 & 148 & 74.0 \\
\hline Not participated & 74 & 37.0 & 84 & 42.0 & 50 & 25.0 & 52 & 26.0 \\
\hline$\chi^{2}$ & \multicolumn{4}{|c|}{$1.04, \mathrm{df}=1, \mathrm{p}=0.30$} & \multicolumn{4}{|c|}{$0.053, \mathrm{df}=1, \mathrm{p}=0.817$} \\
\hline $\mathbf{r}$ & \multicolumn{4}{|c|}{$-0.130, p=0.009$} & \multicolumn{4}{|c|}{$-0.169, p=0.001$} \\
\hline \multicolumn{9}{|l|}{ Frequency of participation } \\
\hline Once & 53 & 42.1 & 38 & 32.8 & 58 & 38.7 & 60 & 40.5 \\
\hline Often & 15 & 11.9 & 9 & 7.8 & 16 & 10.7 & 14 & 9.5 \\
\hline Sometimes & 33 & 26.2 & 37 & 31.9 & 35 & 23.3 & 37 & 25.0 \\
\hline Always & 25 & 19.8 & 32 & 27.6 & 41 & 27.3 & 37 & 25.0 \\
\hline$\chi^{2}$ & \multicolumn{4}{|c|}{$4.65, \mathrm{df}=3, \mathrm{p}=0.19$} & \multicolumn{4}{|c|}{$0.41, \mathrm{df}=3, \mathrm{p}=0.93$} \\
\hline $\mathbf{r}$ & \multicolumn{4}{|c|}{$0.064, p=0.290$} & \multicolumn{4}{|c|}{$-0.076, p=0.217$} \\
\hline \multicolumn{9}{|l|}{ Reason for participation } \\
\hline Innocent killings & 16 & 12.7 & 28 & 24.1 & 36 & 24.0 & 49 & 33.1 \\
\hline Harassment of women & 0 & 0.0 & 13 & 11.2 & 7 & 4.7 & 7 & 4.7 \\
\hline Unjustified arrests of youth & 2 & 1.6 & 15 & 12.9 & 9 & 6.0 & 10 & 6.8 \\
\hline All of the above & 108 & 85.7 & 60 & 51.7 & 98 & 65.3 & 82 & 55.4 \\
\hline$\chi^{2}$ & \multicolumn{4}{|c|}{$39.5, \mathrm{df}=3, \mathrm{p}=0.00$} & \multicolumn{4}{|c|}{$3.45 . d f=3, p=0.32$} \\
\hline $\mathbf{R}$ & \multicolumn{4}{|c|}{$-0.221, p=0.000$} & \multicolumn{4}{|c|}{$-0.013, p=0.831$} \\
\hline
\end{tabular}

Based on field survey

df denotes degree of freedom

$\chi 2$ denotes chi square

$\mathrm{p}$ denotes spearman's level of significance

r denotes spearman's correlation 
Table 4.3 Involvement in Conflict as per Gender

\begin{tabular}{|c|c|c|c|c|c|c|c|c|}
\hline \multirow[t]{3}{*}{ Variable } & \multicolumn{4}{|c|}{ Intact $(\mathrm{N}=400)$} & \multicolumn{4}{|c|}{ Disrupted $(\mathrm{N}=400)$} \\
\hline & \multicolumn{2}{|c|}{ Boys } & \multicolumn{2}{|c|}{ Girls } & \multicolumn{2}{|c|}{ Boys } & \multicolumn{2}{|c|}{ Girls } \\
\hline & $\mathrm{F}$ & $\%$ & $\mathrm{~F}$ & $\%$ & $\mathrm{~F}$ & $\%$ & $\mathrm{~F}$ & $\%$ \\
\hline \multicolumn{9}{|l|}{ Participated in protests } \\
\hline Participated & 141 & 70.5 & 101 & 50.5 & 160 & 80.5 & 138 & 69.0 \\
\hline Not participated & 59 & 29.5 & 99 & 49.5 & 40 & 20.0 & 62 & 31.0 \\
\hline$\chi^{2}$ & \multicolumn{4}{|c|}{$16.7, \mathrm{df}=1, \mathrm{p}=0.00$} & \multicolumn{4}{|c|}{$6.36, \mathrm{df}=1, \mathrm{p}=0.11$} \\
\hline $\mathbf{r}$ & \multicolumn{4}{|c|}{$0.041, p=0.000$} & \multicolumn{4}{|c|}{$0.774, p=0.74$} \\
\hline \multicolumn{9}{|l|}{ Frequency of participation } \\
\hline Once & 45 & 31.9 & 46 & 45.5 & 64 & 40.0 & 54 & 39.1 \\
\hline Often & 8 & 5.7 & 16 & 15.8 & 6 & 3.8 & 24 & 17.4 \\
\hline Sometimes & 38 & 27.0 & 32 & 31.7 & 38 & 23.8 & 34 & 24.6 \\
\hline Always & 50 & 35.5 & 7 & 6.9 & 52 & 32.5 & 26 & 18.8 \\
\hline$\chi^{2}$ & \multicolumn{4}{|c|}{$29.8, \mathrm{df}=3, \mathrm{p}=0.00$} & \multicolumn{4}{|c|}{$19.0, \mathrm{df}=3, \mathrm{p}=0.00$} \\
\hline $\mathbf{r}$ & \multicolumn{4}{|c|}{$0.741, p=0.002$} & \multicolumn{4}{|c|}{$-0.110, p=0.001$} \\
\hline \multicolumn{9}{|l|}{ Reason for participation } \\
\hline Innocent killings & 36 & 25.5 & 8 & 7.9 & 53 & 33.1 & 32 & 23.2 \\
\hline Harassment of women & 0 & 0.0 & 13 & 12.9 & 4 & 2.5 & 10 & 7.2 \\
\hline Unjustified arrests of youth & 16 & 11.3 & 1 & 1.0 & 17 & 10.6 & 2 & 1.4 \\
\hline All of the above & 89 & 63.1 & 79 & 78.2 & 86 & 53.8 & 94 & 68.1 \\
\hline$\chi^{2}$ & \multicolumn{4}{|c|}{$39.1, \mathrm{df}=3, \mathrm{p}=0.00$} & \multicolumn{4}{|c|}{$18.4, \mathrm{df}=3, \mathrm{p}=0.00$} \\
\hline $\mathbf{r}$ & \multicolumn{4}{|c|}{$-0.242, p=0.000$} & \multicolumn{4}{|c|}{$-0.019, p=0.622$} \\
\hline
\end{tabular}

Based on field survey

df denotes degree of freedom

$\chi 2$ denotes chi square

$\mathrm{p}$ denotes spearman's level of significance

r denotes spearman's correlation

\section{CONCLUSION}

More intact adolescents have participated in protests as compared to disrupted adolescents. However, more disrupted rural-urban adolescents have participated in protests than intact rural-urban adolescents. Similarly, more boys have participated than girls. Most intact adolescents always participate in protests. While, majority adolescent girls have participated only once. Reasons put forth by most adolescents for participation in protests include innocent killings, harassment of women and unjustified arrests of youth.

\section{REFERENCES}

1. Amone-P'olak, K.Garnefski, N. and Kraaij. V., (2007) The impact of war experiences and physical abuse on formerly abducted boys in northern Uganda, South African psychiatry review, 10(2):76-82, retrieved from https://journals.co.za/content/medjda/10/2/EJC729 73
2. Bazaz, J., (2016) South Kashmir youth fret loss of momentum, Report: Kashmir reader, retrieved from http://kashmirreader.com/2016/07/27/southkashmir-youth-fret-loss-of-momentum/

3. Betancourt, T. Salhi, S. Buka, S. Leaning, J. Dunn, G. and Earls, F., (2012). Connectedness, social support and internalizing emotional and behavioral problems in adolescents displaced by the Chechen conflict. Disasters journal, 36(4):635655 , retrieved from

http://onlinelibrary.wiley.com/doi/10.1111/j.14677717.2012.01280.x/full

4. Bleasdale, M., (2013). Child soldiers. Report: Human rights watch, retrieved from https://www.hrw.org/topic/childrens-rights/childsoldiers. 
5. Gow, M. Vandergrift, K. and Wanduragala, R. (2000). The right to peace: children and armed conflict, Report: World vision, retrieved from $\mathrm{http} /$ www.worlsvision.com.au/Libraries/3_3_1_C hildren_PDF_reports/The_Right_to_Peace__Children_and_Armed_Conflict.pdf

6. IEP, (2017). Measuring peace in a complex world. Report: institute for Economics and Peace, retrieved from

$\mathrm{http} / /-$

www.visionhumanity.org/app/uploads/2017/06/G

PI-2017-Report-1.pdf

7. Khamis, V., (2012). Impact of war, religiosity and ideology on PTSD and psychiatric disorders in adolescents from Gaza Strip and South Lebanon. Journal of social science and medicine, 74 (12): 2005-2011, retrieved from

http://www.sciencedirect.com/science/article/pii/s o277953612001992.

8. Malik, B., (2016). The role of women in the Kashmir uprising. Report: The Kashmir monitor, retrieved from

http://www.kashmirmonitor.in/Details/108412/the -role-of-women-in-the-kashmir-uprising.

9. Mehta, N., (2016). Kashmir: Why are young people protesting. Report: Al-jazeera, retrieved from

http://www.aljazeera.com/news/2016/07/kashmiryoung-people-protesting-160716201453750.html
10. Mirani, H., (2016). Slogans, protests are 'new plays' for Kashmir. Report: Greater Kashmir, retrieved from

http://www.greaterkashmir.com/news/kashmir/sto ry/228226.html.

11. Mustafa, S., (2016). Your media has wounded the valley': Kashmir youth speaks out. Report: The Citizen is you, retrieved from http://www.thecitizen.in/index.php/NewsDetail/in dex/1/9247/Your-Media-Has-Wounded-theValley-Kashmir-Youth-Speak-Out.

12. Nadimpally, S., (2017). Use of pellet guns has caused a public health crisis in Kashmir. Report: The Wire, retrieved from http//-www.thewirein/119579/pellet-gunskashmir-public.health.

13. Okello, J. Onen, T. Musisi, S., (2007). psychiatric disorders among war-abducted and non-abducted adolescents in Gulu district, Uganda: A comparative study. African journal of psychiatry 10(4):225-231, retrieved from https://journals.co.za/content/medjda2/10/4/EJC72 639

14. Withnall, A., (2014). World peace-There are the only 11 countries in the world that are actually free from conflict. Report: The Independent, retrieved from

http://www.independent.co.uk/news/world/politics /world-peace-these-are-the-only-11-countries-inthe-world-that-are-actually-free-from-conflict9669623.html 